\title{
Electron-Positron Interaction in Metals. Theory and Experiment
}

\author{
H. Stachowiak AND E. BorońsKi \\ W. Trzebiatowski Institute for Low Temperature and Structure Research \\ Polish Academy of Sciences, P.O. Box 1410, 50-950 Wrocław 2, Poland \\ The electron-positron interaction greatly complicates the interpretation \\ of positron annihilation data. The two-detector Doppler measurements of \\ Mijnarends et al. as well as our theoretical calculations point at the con- \\ clusion that the local density approximation to $\mathrm{e}^{+}-\mathrm{e}^{-}$interaction is a good \\ way of treating this problem in real metals, at least the simplest ones. This \\ shows that $\mathrm{e}^{+}-\mathrm{e}^{-}$interaction in an electron gas is the key to understanding \\ this phenomenon also in inhomogeneous systems. On the basis of dozens \\ of experiments one comes to the conclusion that the well known formula \\ of Boroński and Nieminen for the electron accumulation on the positron in \\ jellium describes the best the positron lifetimes in metals. However, it is \\ based on the calculations of Lantto which start from a physically oversim- \\ plified trial function. The results of Arponen and Pajanne, of Rubaszek and \\ Stachowiak, and of Stachowiak and Lach lead to too short positron lifetimes \\ in spite of using less controversial assumptions. The discrepancy is of the \\ order of 8 to $15 \%$ for $r_{\mathrm{s}}=2$. This shows that we still do not fully understand \\ $\mathrm{e}^{+}-\mathrm{e}^{-}$interaction even in an electron gas.
}

PACS numbers: $71.60 .+\mathrm{z}, 78.70 . \mathrm{Bj}$

\section{Introduction}

In our considerations we will start from the following statements:

1. Electron-positron interaction (EPI) increases the electron density on the positron in metals typically by one order of magnitude. The positron lifetime behaves accordingly.

2. Theoretical calculations have been performed mainly for a homogeneous electron gas. What is the usefulness of the results obtained in this way as concerns application to real materials?

3. As well experimental investigations (Mijnarends et al. [1] by two-detector Doppler broadening in $\mathrm{Al}$ ) as our theoretical calculations [2, 3] (cf. also Sect. 9) 
point at the local density approximation (LDA) of $\mathrm{e}^{+}-\mathrm{e}^{-}$correlations as a reasonable way of applying jellium results to real materials.

4. The above circumstances make it at last possible to find how well theoretical calculations performed for jellium are confirmed by experimental measurements of positron lifetime in real materials.

\section{Theoretical approaches to $\mathrm{e}^{+}-\mathrm{e}^{-}$interaction in jellium}

We will concentrate on the following approaches (for more details cf. [4]):

1. Calculations basing on the Bethe-Goldstone equation (Kahana [5], Carbotte [6, 7], Rubaszek and Stachowiak [8], Sormann [9]).

2. The approach of Arponen and Pajanne [10] in which the Hamiltonian is transcribed in terms of the Sawada bosons.

The remaining approaches base on the theory of liquids.

3. Lantto assumes a Jastrow type trial function and performs minimization of the Hamiltonian using the Fermi hypernetted-chain approximation (FHNC) [11].

4. Starting from the hypernetted-chain (HNC) results of Lantto and coworkers (Kallio, Pietiläinen) [12, 13] Gondzik and Stachowiak [14] propose an approach sufficiently simple to be applied directly to real materials [3, 2, 15].

5. The perturbed HNC (PHNC) approach of Stachowiak and Lach $[16,17]$ provides an alternative way to study the effect of $\mathrm{e}^{+}-\mathrm{e}^{-}$correlations in jellium.

\section{The wave function of the system}

The usefulness of positron annihilation for studies of the electronic structure is based on conservation of the identity of electronic states during the interaction with the positron (this point of view is supported by the theoretical result of Majumdar that $\mathrm{e}^{+}-\mathrm{e}^{-}$interaction does not affect the Fermi surface [18]). For this reason the wave function of the jellium-one positron system is assumed as a Slater determinant built of functions $\psi_{\boldsymbol{k} \sigma}\left(\boldsymbol{r}_{\mathrm{e}}, \boldsymbol{r}_{\mathrm{p}}\right)$. The functions $\psi_{\boldsymbol{k}}$ (let us omit the spin index) describe the scattering of the electron plane wave on the screened positron and obey the equation (in the Hartree atomic units)

$$
\left[-\frac{1}{2} \nabla_{\mathrm{e}}^{2}-\frac{1}{2} \nabla_{\mathrm{p}}^{2}+V\left(\left|\boldsymbol{r}_{\mathrm{e}}-\boldsymbol{r}_{\mathrm{p}}\right|\right)\right] \psi_{\boldsymbol{k}}=\frac{k^{2}}{2} \psi_{\boldsymbol{k}} .
$$

Describing the $\mathrm{e}^{+}-\mathrm{e}^{-}$interaction in this way is, of course, an approximation, but attempts to go beyond this approximation led in our opinion to very few conclusive results. However, we consider this problem as important.

The most general form of $\psi_{\boldsymbol{k}}(\boldsymbol{s})$ can be written as

$$
\psi_{\boldsymbol{k}}(\boldsymbol{s})=\frac{1}{\sqrt{\Omega}} w(s)\left[\mathrm{e}^{\mathrm{i} \boldsymbol{k} \boldsymbol{s}}+v_{\boldsymbol{k}}(\boldsymbol{s})\right],
$$

where $s=\boldsymbol{r}_{\mathrm{e}}-\boldsymbol{r}_{\mathrm{p}}$. 


\section{The density of the screening cloud}

The most important quantity to compute is the density of the screening cloud. Since the functions $\psi_{\boldsymbol{k}}$ are weakly nonorthogonal, we will obtain a better approximation taking this into account.

The general formula for the electron density is

$$
\rho\left(\boldsymbol{s}_{1}\right)=N \frac{\int|\Psi|^{2} \mathrm{~d} \tau_{1}}{\int|\Psi|^{2} \mathrm{~d} \tau}
$$

where

$$
\boldsymbol{s}_{i}=\boldsymbol{r}_{i}-\boldsymbol{r}_{\mathrm{p}}, \quad \mathrm{d} \tau=\prod_{i=1}^{N} \mathrm{~d} \boldsymbol{s}_{\mathrm{i}}, \quad \mathrm{d} \tau_{1}=\frac{\mathrm{d} \tau}{\boldsymbol{s}_{1}} .
$$

$\Psi$ is the wave function of the system expressed by the Slater determinant. $N$ is the number of electrons, $\boldsymbol{r}_{i}$ are coordinates of the $i$ electron.

In the case of weak nonorthogonality of the wave functions $\psi_{\boldsymbol{k}}$, we get from (3) the formula for the electron density

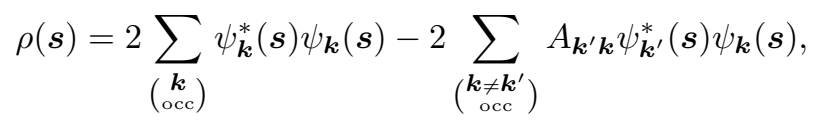

where

$$
A_{\boldsymbol{k}^{\prime} \boldsymbol{k}}=\int \mathrm{d} \boldsymbol{s}^{\prime} \psi_{\boldsymbol{k}}^{*}\left(\boldsymbol{s}^{\prime}\right) \psi_{\boldsymbol{k}^{\prime}}\left(\boldsymbol{s}^{\prime}\right)
$$

\section{Application of the Bethe-Goldstone equation}

\subsection{The Kahana approach}

The wave function $\psi_{\boldsymbol{k}}$ is assumed in the form

$$
\psi_{\boldsymbol{k}}(\boldsymbol{s})=\frac{1}{\sqrt{\Omega}}\left[\mathrm{e}^{\mathrm{i} \boldsymbol{k} s}+v_{\boldsymbol{k}}(\boldsymbol{s})-v_{\boldsymbol{k}}^{\prime}(\boldsymbol{s})\right]
$$

where

$$
v_{\boldsymbol{k}}(\boldsymbol{s})=\frac{1}{\Omega} \sum_{\boldsymbol{q}} C_{\boldsymbol{k}}(\boldsymbol{q}) \mathrm{e}^{\mathrm{i}(\boldsymbol{k}+\boldsymbol{q}) \boldsymbol{s}}
$$

and

$$
v_{\boldsymbol{k}}^{\prime}(\boldsymbol{s})=\frac{1}{\Omega} \sum_{\substack{\boldsymbol{q} \\|\boldsymbol{k}+\boldsymbol{q}|<k_{\mathrm{F}}}} C_{\boldsymbol{k}}(\boldsymbol{q}) \mathrm{e}^{\mathrm{i}(\boldsymbol{k}+\boldsymbol{q}) \boldsymbol{s}} .
$$

$C_{\boldsymbol{k}}(\boldsymbol{q})$ are Fourier coefficients.

In this way scattering to momentum states inside the Fermi surface is excluded. $\psi_{\boldsymbol{k}}$ is obtained under 4 assumptions:

1. The effective potential of $\mathrm{e}^{+}-\mathrm{e}^{-}$interaction is the static random phase approximation (RPA) potential. 
2. The electrons can scatter only to momentum states outside the Fermi sphere, since the states inside the Fermi sphere are fully occupied.

3. The scattering does not depend on the angle between $\boldsymbol{k}$ and $\boldsymbol{k}+\boldsymbol{q}$ (this is a rather controversial simplification).

4. The electron density is computed from the usual formula

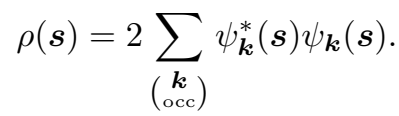

Under these conditions Eq. (1) takes the form of a Bethe-Goldstone equation for the Fourier coefficients $\chi(\boldsymbol{k}, \boldsymbol{p})$ of the wave function $\psi_{\boldsymbol{k}}$ :

$$
\begin{aligned}
& \chi(\boldsymbol{k}, \boldsymbol{p})=\frac{a V(|\boldsymbol{k}-\boldsymbol{p}|)}{p^{2}+(\boldsymbol{k}-\boldsymbol{p})^{2}-k^{2}} \\
& \quad+\frac{a}{p^{2}+(\boldsymbol{k}-\boldsymbol{p})^{2}-k^{2}} \int_{q \geq 1} \mathrm{~d} \boldsymbol{q} V(|\boldsymbol{q}-\boldsymbol{p}|) \chi(\boldsymbol{q}, \boldsymbol{p}),
\end{aligned}
$$

where momenta are expressed in units of the Fermi momentum $k_{\mathrm{F}}, a=2 /\left(8 \pi^{3} k_{\mathrm{F}}\right)$ and $V(q)$ are the Fourier transforms of the effective electron-positron potential. This equation has been derived by Kahana (Eq. (24) in [5], cf. also [19]).

The second assumption is not very convincing and has been criticized. In the case of screening a heavy particle the states rearrange. Instead of describing them with 3 quantum numbers $k_{x}, k_{y}, k_{z}$ corresponding to the representation in Cartesian coordinates we describe them with the quantum numbers $n, l$, and $m$, characteristic of spherical coordinates. The second assumption needs not to be introduced. In general, the principles of physics impose the antisymmetry of the wave function of the system. The Pauli exclusion principle is just a consequence of this property. In the case of a positron using the wave function (2) shows that the second assumption is too restrictive. The effect of the second assumption will depend on the shape of $w(s)$.

\subsection{The approach of Rubaszek and Stachowiak}

It differs from Kahana by omitting from the third assumption and by applying the Kohn-Sham potential in the Bethe-Goldstone equation [5] (instead of the RPA potential). This potential is computed self-consistently and has the form

$$
V(\boldsymbol{s})=-\frac{1}{s}+\int \mathrm{d} \boldsymbol{s}^{\prime} \frac{\rho\left(s^{\prime}\right)-\rho_{0}}{\left|\boldsymbol{s}-\boldsymbol{s}^{\prime}\right|}+V_{\mathrm{HL}}[\rho(s)]-V_{\mathrm{HL}}\left(\rho_{0}\right),
$$

where $V_{\mathrm{HL}}(\rho)$ is the exchange-correlation correction chosen e.g. in the form proposed by Hedin and Lundqvist. $\rho_{0}$ is the density of the electron gas.

This approach removes some of the difficulties encountered by the approach of Kahana. In particular, non-physical properties of the annihilation rates of Kahana (low density divergence of the annihilation rates) are avoided. This is due both to achieving self-consistency and to including the exchange-correlation correction in the potential (Fig. 1). 


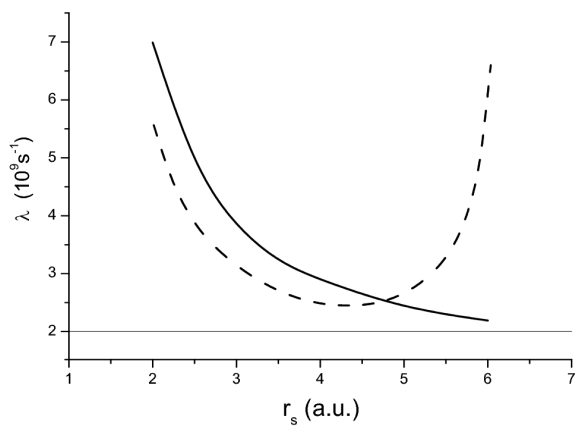

Fig. 1. Annihilation rates of Kahana [5] (dashed curve) and of Rubaszek and Stachowiak [8] (full curve).

\section{Approaches based on the theory of liquids}

The theory of liquids is a field of theoretical physics intensively developed since the fifties [20]. The simplest case of this theory concerns a classical liquid. Its development led to the elaboration of two particularly useful approximations called Percus-Yevick and HNC. The first one, though being less satisfactory from the point of view of the number of terms included in the diagram expansion, leads to better results in the case when the particles of the liquid possess a hard core. This is not the case of electrons. In their case it is preferable to use the HNC approximation.

Formal mathematical similarity allows to extend the results of the classical theory to the quantum case. However, while in the classical case the solution of the problem provided directly the equation of state, in the quantum case it only helps to compute the average value of the Hamiltonian while the system consists of bosons and the trial function of the system is assumed in a special form called the Jastrow trial function. In the case of fermions the HNC approximation appears in the more complicated form of the Fermi HNC.

\subsection{Calculations of Lantto}

These calculations basing faithfully on a Jastrow type trial function are self-consistent. In the case of a system of one positron and $N$ electrons the trial function of Lantto has the form

$$
\Psi=F_{12} F_{11} \Phi,
$$

where the factors $F_{11}$ and $F_{12}$ are expressed by the functions-parameters $f_{11}$ and $f_{12}$ through the formulae

$$
F_{11}=\prod_{i j} f_{11}\left(\boldsymbol{r}_{i}, \boldsymbol{r}_{j}\right), \quad F_{12}=\prod_{i} f_{12}\left(\boldsymbol{r}_{i}, \boldsymbol{r}_{\mathrm{p}}\right) .
$$


$\Phi$ is the Slater determinant of free electrons. It is obvious that the variational function-parameter $f_{11}$ offers no possibility to minimize the Hamiltonian with regard to the dependence of $\mathrm{e}^{-}-\mathrm{e}^{-}$correlations on the distance from the positron. Also the function-parameter $f_{12}$ does not include the important momentum dependence of electron scattering on the positron. This is why the momentum dependence of the annihilation probability cannot be obtained in this formalism. The calculations have been performed using the Fermi HNC approach in all its complexity.

The results of Lantto have been parameterized by Boroński and Nieminen [21], including additionally some other physical requirements. Recent calculations using the Monte Carlo approach failed to reproduce the numerical results of Lantto [22].

\subsection{Approach of the Oulu group}

Kallio et al. adopting after Zabolitzky [23] the Lado approximation, proposed a particularly simple way of minimizing the trial function $(13)[12,13]$. This resulted in the equation

$$
\left[-\nabla^{2}+V(s)\right] w(s)=0
$$

for the electron density amplitude $w(s)$. $V(s)$ remained, however, a rather complicated functional of the electron distribution.

\subsection{Approach of Gondzik and Stachowiak}

The complexity of the potential $V(s)$ in Eq. (15) made it difficult to achieve self-consistency, at least for low values of the electron density. Moreover this potential, anyway, was not very reliable, because of poor account of the electron-electron correlations in the trial function (13). This is why Gondzik and Stachowiak proposed to simply replace this potential by its Kohn-Sham form [14]

$$
V_{0}(\boldsymbol{s})=-\frac{1}{s}+\int \mathrm{d} \boldsymbol{s}^{\prime} \frac{w^{2}\left(s^{\prime}\right)-\rho_{0}}{\left|\boldsymbol{s}-\boldsymbol{s}^{\prime}\right|}+V_{\mathrm{HL}}\left[w^{2}(s)\right]-V_{\mathrm{HL}}\left(\rho_{0}\right) .
$$

The computation of the positron annihilation rate in the above formalism is particularly simple and consists in solving a single nonlinear integro-differential equation. The results are reasonable (cf. Fig. 2) and easy to obtain in the whole range of electron densities.

\subsection{The perturbed HNC approach}

The second assumption of Kahana is controversial, since the Pauli exclusion principle should follow from the antisymmetry of the wave function $\Psi$ and not from any additional assumption.

Let us note that when the form (2.4) of Ref. [16] is adopted for $\psi_{\boldsymbol{k}}$ the wave function of the system can be written as 


$$
\Psi\left(\boldsymbol{s}_{1} \ldots \boldsymbol{s}_{N}\right)=\prod_{i=1}^{N} w\left(s_{i}\right) \Phi\left(\boldsymbol{s}_{1} \ldots \boldsymbol{s}_{N}\right),
$$

where $\Phi$ is a Slater determinant built from functions

$$
\varphi_{\boldsymbol{k}}(\boldsymbol{s})=\frac{1}{\sqrt{\Omega}}\left[\mathrm{e}^{\mathrm{i} \boldsymbol{k} \boldsymbol{s}}+v_{\boldsymbol{k}}(\boldsymbol{s})\right] .
$$

In Refs. [16] and [17] it was assumed that the form (17) of the wave function where $w(s)$ is obtained by solving the Gondzik-Stachowiak equation

$$
\left[-\nabla^{2}+V_{0}(\boldsymbol{s})\right] w(\boldsymbol{s})=0
$$

allows $v_{\boldsymbol{k}}$ to be small, so terms quadratic in $v_{\boldsymbol{k}}$ can be omitted. This made possible to solve Eqs. (1) self-consistently.

\section{The approach of Arponen and Pajanne}

A satisfactory theory of EPI should include three necessary features, namely self-consistency, momentum dependence of electron-positron scattering and realistic account of electron-electron correlations. These features are included in the approach of Rubaszek and Stachowiak to the Bethe-Goldstone formalism and in PHNC. They are also included in the approach of Arponen and Pajanne (AP). They are not included in the approach of Lantto nor in that of Kahana.

The AP approach bases on a completely different formalism than the approaches presented above. For a pure electron gas the Hamiltonian of second quantization has the form

$$
\hat{H}_{\mathrm{e}}=\sum_{\boldsymbol{p} \sigma} \varepsilon_{\boldsymbol{p}} c_{\boldsymbol{p} \sigma}^{+} c_{\boldsymbol{p} \sigma}+\frac{1}{2 \Omega} \sum_{\boldsymbol{q}}^{\prime} v_{\boldsymbol{q}}\left(\rho_{-\boldsymbol{q}} \rho_{\boldsymbol{q}}-\hat{N}_{\mathrm{e}}\right),
$$

where $c_{\boldsymbol{p} \sigma}^{+}$and $c_{\boldsymbol{p} \sigma}$ are electron creation and annihilation operators,

$$
\begin{aligned}
\rho_{\boldsymbol{q}} & =\sum_{\boldsymbol{p} \sigma} c_{\boldsymbol{p} \sigma}^{+} c_{\boldsymbol{p}+\boldsymbol{q} \sigma}, \\
v_{\boldsymbol{q}} & =\frac{e^{2}}{q^{2}}
\end{aligned}
$$

$\hat{N}_{\mathrm{e}}$ is the electron number operator. The prime in the summation means that $\boldsymbol{q}=\mathbf{0}$ is excluded.

The approach of AP is based on the idea of Sawada and Wentzel of expressing $\hat{H}_{\mathrm{e}}$ through boson-like operators. A part of $\hat{H}_{\mathrm{e}}$ is separated out and called RPA Hamiltonian

$$
\hat{H}_{\mathrm{e}}^{\mathrm{RPA}}=\sum_{\boldsymbol{p} \sigma} \varepsilon_{\boldsymbol{p}} c_{\boldsymbol{p} \sigma}^{+} c_{\boldsymbol{p} \sigma}+\sum_{\boldsymbol{q}}^{\prime} v_{\boldsymbol{q}} \sigma_{-\boldsymbol{q}} \sigma_{\boldsymbol{q}}
$$

where

$$
\sigma_{\boldsymbol{q}}=\sum_{\boldsymbol{p} \sigma}[1-\Delta(\boldsymbol{p}, \boldsymbol{p}+\boldsymbol{q})] c_{\boldsymbol{p} \sigma}^{+} c_{\boldsymbol{p}+\boldsymbol{q} \sigma} .
$$


Here $\Delta\left(\boldsymbol{p}, \boldsymbol{p}^{\prime}\right)=1$ if $\boldsymbol{p}$ and $\boldsymbol{p}^{\prime}$ are both greater or smaller than $k_{\mathrm{F}}$ and zero otherwise. This Hamiltonian can be approximately diagonalized by introducing the boson-like operators $A_{\boldsymbol{q} E}$.

In the Sawada boson representation the RPA Hamiltonian becomes

$$
\hat{H}_{\mathrm{e}}^{\mathrm{RPA}}=\sum_{\boldsymbol{q} E} E A_{\boldsymbol{q} E}^{+} A_{\boldsymbol{q} E} .
$$

The boson vacuum corresponds to the ground RPA state of the electron gas. Solving the problem of $\mathrm{e}^{+}-\mathrm{e}^{-}$interaction in jellium consists roughly in computing the distribution of the Sawada bosons in presence of the positron.

\section{The annihilation rates}

The annihilation rate (inverse of lifetime) is one of the basic quantities characterizing positron annihilation in the material under investigation. Theoretical results obtained for an electron gas can be applied with a certain degree of reliability only to metallic materials. We will not deal here with situations where several lifetimes are observed.

As concerns the homogeneous electron gas, the annihilation rate $\lambda\left(r_{\mathrm{s}}\right)$ is obtained from the formula

$$
\lambda\left(r_{\mathrm{s}}\right)=\frac{12}{r_{\mathrm{s}}^{3}} g\left(r_{\mathrm{s}}, 0\right) \times 10^{9} / \mathrm{s} .
$$

$g\left(r_{\mathrm{s}}, r\right)$ is the electron-positron correlation factor. For $r=0$ it gives information about the accumulation of electrons on the positron. It is equal to 1 in case when the $\mathrm{e}^{+}-\mathrm{e}^{-}$interaction is neglected.

Several authors proposed on basis of theoretical calculations different formulae for the correlation factors. For example Boroński and Nieminen (BN) [21] using mainly the results of Lantto [12] wrote the formula

$$
g\left(r_{\mathrm{s}}, 0\right)=1+1.23 r_{\mathrm{s}}+0.8295 r_{\mathrm{s}}^{3 / 2}-1.26 r_{\mathrm{s}}^{2}+0.3286 r_{\mathrm{s}}^{5 / 2}+r_{\mathrm{s}}^{3} / 6 .
$$

The results of AP [10] led to a correlation factor obeying the formula proposed by Barbiellini et al. [24]

$$
g\left(r_{\mathrm{s}}, 0\right)=1+1.23 r_{\mathrm{s}}-0.0742 r_{\mathrm{s}}^{2}+r_{\mathrm{s}}^{3} / 6
$$

Stachowiak and Lach (SL) [17] on basis of PHNC results proposed the form

$$
g\left(r_{\mathrm{s}}, 0\right)=1+1.23 r_{\mathrm{s}}-0.1375 r_{\mathrm{s}}^{2}+r_{\mathrm{s}}^{3} / 6
$$

It is worth mentioning that the results of Rubaszek and Stachowiak [8] and of Lowy and Jackson [25] do not differ in principle from those of AP. The results of Gondzik and Stachowiak [14] agree with the formula

$$
\begin{array}{r}
g\left(r_{\mathrm{s}}, 0\right)=1.01906+1.33696 r_{\mathrm{s}}+0.13651 r_{\mathrm{s}}^{2}+0.08112 r_{\mathrm{s}}^{3} \\
\quad+0.00863 r_{\mathrm{s}}^{4}-3.2491 \times 10^{-4} r_{\mathrm{s}}^{5}+4.41454 \times 10^{-6} r_{\mathrm{s}}^{6} .
\end{array}
$$


The annihilation rate described by the formulae (27)-(30) are shown in Fig. 2. The results of applying them to real metals are collected in Table.

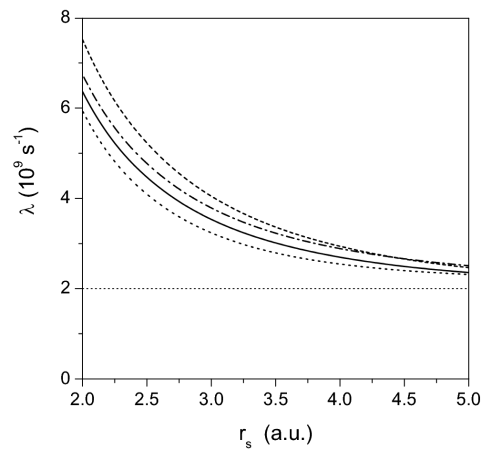

Fig. 2. Comparison of different formulae for the positron annihilation rate in an electron gas (dashed curve - HNC [14], dashed-dotted curve - Barbiellini et al. [24], solid curve - PHNC [16], dotted curve - Boroński-Nieminen [21]).

TABLE

Positron lifetimes (in ps) in metals from the first and second group, and for Al. The experimental values [26] are given in the second column. Some more recent experimental data [27] are labeled with an asterisk. The next three columns present the results of LMTO-ASA calculations of Rubaszek et al. [28] (from Table V in Ref. [28]). The next six columns present our FLAPW results. Let us note that the 9 th, 10th and 11th columns contain the results of calculations [15] taking into account the smaller enhancement due to the nonzero momentum of the positron. The last column presents the contribution (in \%) of the higher components in the Fourier expansion of the positron wave function of the corresponding metals.

\begin{tabular}{|c|c|c|c|c|c|c|c|c|c|c|c|}
\hline \multirow[t]{2}{*}{ Metal } & \multirow[t]{2}{*}{ Exp. } & \multicolumn{3}{|c|}{$\begin{array}{c}\text { LMTO-LDA } \\
\text { (Ref. [28]) }\end{array}$} & \multicolumn{3}{|c|}{$\begin{array}{c}\text { FLAPW- LDA } \\
\text { (Ref. [15]) }\end{array}$} & \multicolumn{3}{|c|}{$\begin{array}{l}\text { Heavy positron } \\
\quad \text { (Ref. [15]) }\end{array}$} & \multirow[t]{2}{*}{$\begin{array}{l}\% \text { high } \\
\text { comp. }\end{array}$} \\
\hline & & $\mathrm{BN}$ & $\mathrm{SL}$ & $\mathrm{AP}$ & $\mathrm{BN}$ & $\mathrm{SL}$ & $\mathrm{AP}$ & $\mathrm{BN}$ & $\mathrm{SL}$ & $\mathrm{AP}$ & \\
\hline $\mathrm{Li}$ & 291 & 300 & 284 & 260 & 298 & 275 & 258 & 300 & 277 & 260 & 1.7 \\
\hline $\mathrm{Na}$ & 338 & 328 & 323 & 291 & 328 & 308 & 294 & 332 & 312 & 294 & 2.7 \\
\hline K & 397 & 367 & 373 & 331 & 368 & 352 & 332 & 375 & 359 & 338 & 4.2 \\
\hline $\mathrm{Rb}$ & 406 & 377 & 388 & 342 & 377 & 364 & 343 & 385 & 372 & 351 & 4.6 \\
\hline Cs & 418 & 389 & 409 & 357 & 388 & 377 & 355 & 398 & 386 & 364 & 6.5 \\
\hline $\mathrm{Al}$ & 163 & 163.4 & 155.4 & 145 & 162.3 & 152 & 143.9 & 165.9 & 155.3 & 147.2 & 5.3 \\
\hline $\mathrm{Cu}$ & $\begin{array}{l}110 \\
118^{*}\end{array}$ & 104.6 & 102.9 & 97 & 105 & 101 & 97.4 & 107.8 & 103.4 & 101 & 5.6 \\
\hline $\mathrm{Ag}$ & $\begin{array}{l}131 \\
136^{*}\end{array}$ & 119.1 & 116.6 & 109 & 121 & 115 & 111 & 126 & 120 & 116 & 7.4 \\
\hline $\mathrm{Au}$ & 117 & 106.0 & 104.2 & 98 & 110 & 105 & 101 & 115 & 110 & 106 & 8.7 \\
\hline
\end{tabular}




\section{Calculation of $\mathrm{e}^{+}-\mathrm{e}^{-}$interaction in lithium}

In absence of the positron, following the approach of Gondzik and Stachowiak, the electronic structure is described by the equations $[2,3]$

$$
\left[-\frac{1}{2} \nabla^{2}+V(\boldsymbol{r})\right] \psi_{1}(\boldsymbol{r})=E_{1} \psi_{1}(\boldsymbol{r}), \quad\left[-\frac{1}{2} \nabla^{2}+V(\boldsymbol{r})\right] \psi_{2}(\boldsymbol{r})=0,
$$

where

$$
\begin{aligned}
V(\boldsymbol{r}) & =-\frac{Z}{r}+2 \int \mathrm{d} \boldsymbol{r}^{\prime} \frac{\psi_{1}^{2}\left(\boldsymbol{r}^{\prime}\right)}{\left|\boldsymbol{r}-\boldsymbol{r}^{\prime}\right|}+\int \mathrm{d} \boldsymbol{r}^{\prime} \frac{\psi_{2}^{2}\left(\boldsymbol{r}^{\prime}\right)-d\left(\boldsymbol{r}^{\prime}\right)}{\left|\boldsymbol{r}-\boldsymbol{r}^{\prime}\right|} \\
& +V_{\mathrm{HL}}\left[2 \psi_{1}^{2}(\boldsymbol{r})+\psi_{2}^{2}(\boldsymbol{r})\right]-V_{\mathrm{HL}}\left(\rho_{0}\right) .
\end{aligned}
$$

$Z$ is the charge of the nucleus, $d(\boldsymbol{r})$ is the distribution of the positive charge in the electron gas. It is assumed in the form

$$
d(r)=\left\{\begin{array}{ccc}
\rho_{0} & \text { for } \quad r>R_{\mathrm{WS}}, \\
0 & \text { for } \quad r<R_{\mathrm{WS}}
\end{array}\right.
$$

where

$$
\rho_{0}=D \frac{3(Z-2)}{4 \pi\left(R_{\mathrm{WS}}\right)^{3}} .
$$

$R_{\mathrm{WS}}$ is the radius of the Wigner-Seitz sphere. $\psi_{1}$ is the wave function of core electrons. This limits us to elements having a two-electron core. $\psi_{2}$ is the density amplitude for conduction electrons. The Lagrange multiplier $E_{2}$ which should occur on the right-hand side of the second equation (31) is normalized to zero by the last term in the formula (32), while $E_{1}$ is the energy eigenstate of core electrons. $D$ differs from unity (and is equal to 1.24 in the case of lithium) because of the necessity to normalize the density of valence electrons within the Wigner-Seitz sphere.

In presence of the positron the above equations take the form

$$
\begin{aligned}
& {\left[-\frac{1}{2} \nabla^{2}+V(r)+\frac{1}{2} W\left(\boldsymbol{r}_{\mathrm{p}}, \boldsymbol{r}\right)\right] \chi_{1}\left(\boldsymbol{r}_{\mathrm{p}}, \boldsymbol{r}\right)=E_{1}\left(r_{\mathrm{p}}\right) \chi_{1}\left(\boldsymbol{r}_{\mathrm{p}}, \boldsymbol{r}\right),} \\
& {\left[-\frac{1}{2} \nabla^{2}+V(r)+\frac{1}{2} W\left(\boldsymbol{r}_{\mathrm{p}}, \boldsymbol{r}\right)\right] \chi_{2}\left(\boldsymbol{r}_{p}, \boldsymbol{r}\right)=0,}
\end{aligned}
$$

where the screened electron-positron potential $W\left(\boldsymbol{r}_{\mathrm{p}}, \boldsymbol{r}\right)$ is defined as

$$
W\left(\boldsymbol{r}_{\mathrm{p}}, \boldsymbol{r}\right)=-\frac{1}{\left|\boldsymbol{r}-\boldsymbol{r}_{\mathrm{p}}\right|}+W_{\mathrm{p}}\left(\boldsymbol{r}_{\mathrm{p}}, \boldsymbol{r}\right)+W_{\mathrm{xc}}\left(\boldsymbol{r}_{\mathrm{p}}, \boldsymbol{r}\right) .
$$

Here

$$
W_{\mathrm{p}}\left(\boldsymbol{r}_{\mathrm{p}}, \boldsymbol{r}\right)=2 \int \mathrm{d} \boldsymbol{r}^{\prime} \frac{\chi_{1}^{2}\left(\boldsymbol{r}_{\mathrm{p}}, \boldsymbol{r}^{\prime}\right)-\psi_{1}^{2}\left(\boldsymbol{r}^{\prime}\right)}{\left|\boldsymbol{r}-\boldsymbol{r}^{\prime}\right|}+\int \mathrm{d} \boldsymbol{r}^{\prime} \frac{\chi_{2}^{2}\left(\boldsymbol{r}_{\mathrm{p}}, \boldsymbol{r}^{\prime}\right)-\psi_{2}^{2}\left(\boldsymbol{r}^{\prime}\right)}{\left|\boldsymbol{r}-\boldsymbol{r}^{\prime}\right|},
$$




$$
W_{\mathrm{xc}}\left(\boldsymbol{r}_{\mathrm{p}}, \boldsymbol{r}\right)=V_{\mathrm{HL}}\left[2 \chi_{1}^{2}\left(\boldsymbol{r}_{\mathrm{p}}, \boldsymbol{r}\right)+\chi_{2}^{2}\left(\boldsymbol{r}_{\mathrm{p}}, \boldsymbol{r}\right)\right]-V_{\mathrm{HL}}\left[2 \psi_{1}^{2}(\boldsymbol{r})+\psi_{2}^{2}(\boldsymbol{r})\right] .
$$

$\chi_{i}$ indicate the form of the functions $\psi_{i}$ in presence of the positron. The positron distribution is described, of course, by the positron wave function.

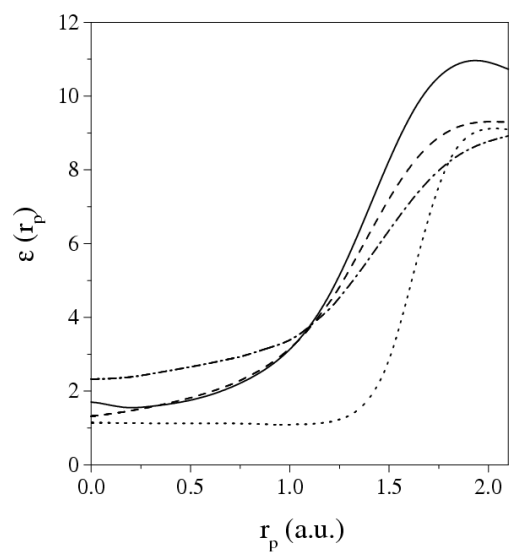

Fig. 3. Effective enhancement $\varepsilon\left(r_{\mathrm{p}}\right)$ inside an atom of lithium embedded in an electron gas calculated in [15] (solid curve) and according to the LDA (dashed curve), the method proposed by Barbiellini et al. (the generalized gradient approximation) [24] (dotted curve) and the method proposed by Rubaszek (the weighted density approximation for the systems containing a positron) [28] (dashed-dotted curve).

The annihilation rates following from solving Eqs. (35) are shown in Fig. 3. Their agreement with the predictions of LDA is striking, at least in the core region. As concerns the interstitial space, in order to obtain reliable results we should use a more realistic function $d(\boldsymbol{r})$ in Eq. (32).

\section{Conclusions}

Calculations have been performed for several metals using different formulae for the $\mathrm{e}^{+}-\mathrm{e}^{-}$correlation function. In each case the LDA approximation to $\mathrm{e}^{+}-\mathrm{e}^{-}$correlations was assumed. The results are shown in Table. Our calculations were performed using the numerical code WIEN95 [29] which uses the full potential linearized augmented plane wave (FLAPW) approach to band structure calculations. The calculations of Rubaszek et al. [28] shown for comparison were performed within the linearized muffin-tin orbital (LMTO) approach.

It is visible that the formula of Boroński and Nieminen gives the best agreement with the experiment. However, it is based on the controversial calculations of Lantto [11]. The more reliable calculations (described in this article) lead to too high positron annihilation rates. There is a need to find the reason for this discrepancy. 


\section{References}

[1] P.E. Mijnarends, A.C. Kruseman, A. van Veen, H. Schut, A. Bansil, J. Phys. Condens. Matter 10, 10383 (1998).

[2] H. Stachowiak, E. Boroński, G. Banach, Mater. Sci. Forum 363-365, 615 (2001).

[3] H. Stachowiak, E. Boroński, http://aps.arxiv.org/abs/cond-mat/0402478, to be published.

[4] H. Stachowiak, A. Rubaszek, in: Positrons at Metallic Surfaces, Solid State Phenomena, Vol. 28-29, Ed. A. Ishii, Trans. Tech. Publ., Aedermannsdorf 1993, p. 7.

[5] S. Kahana, Phys. Rev. 129, 1622 (1963).

[6] J.P. Carbotte, S. Kahana, Phys. Rev. 139, A213 (1965).

[7] Positron Solid State Physics, Eds. W. Brandt, A. Dupasquier, North-Holland, Amsterdam 1983.

[8] A. Rubaszek, H. Stachowiak, Phys. Rev. B 38, 3846 (1988).

[9] H. Sormann, Phys. Rev. B 54, 4558 (1996).

[10] J. Arponen, E. Pajanne, Ann. Phys.(N.Y.) 121, 343 (1979); J. Arponen, E. Pajanne, J. Phys. F 9, 2359 (1979).

[11] L. Lantto, Phys. Rev. B 36, 5160 (1987).

[12] A. Kallio, P. Pietiläinen, L. Lantto, Phys. Scr. 25, 943 (1982); L. Lantto, private communication.

[13] P. Pietiläinen, A. Kallio, Phys. Rev. B 27, 224 (1983).

[14] J. Gondzik, H. Stachowiak, J. Phys. C 18, 5399 (1985).

[15] H. Stachowiak, E. Boroński, in: Proc. 34 Polish Seminar on Positron Annihilation, Turawa (Poland) 2002, Ed. K. Jerie, University of Opole, University of Wrocław, Opole 2002, p. 75.

[16] H. Stachowiak, Phys. Rev. B 41, 12522 (1990).

[17] H. Stachowiak, J. Lach, Phys. Rev. B 48, 9828 (1993).

[18] C.K. Majumdar, Phys. Rev. 140, A227 (1965).

[19] A. Klein, R.E. Prange, Phys. Rev. 112, 1008 (1958).

[20] R. Balescu, Equilibrium and Nonequilibrium Statistical Mechanics, Wiley, New York 1975.

[21] E. Boroński, R.M. Nieminen, Phys. Rev. B 34, 3820 (1986).

[22] L.M. Fraser, Ph.D. thesis, The Blackett Laboratory, University of London, 1995 (www.sst.ph.ic.ac.uk/research/theses/L.M.Fraser.pdf).

[23] J.G. Zabolitzky, Phys. Rev. B 22, 2353 (1980).

[24] B. Barbiellini, M.J. Puska, T. Torsti, R.M. Nieminen, Phys. Rev. B 51, 7341 (1995); B. Barbiellini, M.J. Puska, T. Korhonen, A. Harju, T. Torsti, R.M. Nieminen, Phys. Rev. B 53, 16201 (1996).

[25] D.N. Lowy, A.D. Jackson, Phys. Rev. B 12, 1689 (1975); D.N. Lowy, Phys. Rev. 26, 60 (1982).

[26] A. Seeger, F. Banhart, W. Bauer, in: Positron Annihilation, Eds. L. DorikensVanpraet, M. Dorikens, D. Segers, World Scientific, Singapore 1989, p. 275. 
[27] A. Baranowski, E. Dȩbowska, Appl. Phys. A 51, 23 (1990).

[28] A. Rubaszek, Z. Szotek, W.M. Temmerman, Phys. Rev. B 58, 11285 (1998).

[29] P. Blaha, K. Schwarz, P. Dufek, R. Augustyn, WIEN95, Technical University of Vienna, 1995 (improved and updated Unix version of the original copyrighted WIEN-code, which was published by P. Blaha, K. Schwarz, P. Sorantin, S.B. Trickey, in Comput. Phys. Commun. 59, 399 (1990)). 